\title{
The morphological mismatch changes and adapts after lung transplantation in the patient with Kartagener syndrome
}

\author{
Do Hyung Kim, Chan Hum Kim
}

Department of Thoracic and Cardiovascular Surgery, Pusan National University Yangsan Hospital, Yangsan, Korea

Background: Kartagener syndrome (KS) is a very rare disease with an incidence of one in 20,000 to 30,000 births. When progressing to end-stage KS, lung transplantation is the only treatment option. Because the patient with KS has anatomical abnormalities such as situs inversus totalis. Surgery should be performed with anatomical problem. We underwent lung transplantation in the patient with KS and observed the morphological adaptation for 6 months through computed tomography (CT) to find out how the morphological mismatch changes and adapts.

Methods: A 54-year-old male patient with KS underwent bilateral LT with donor's lungs from a 37-year-old female who died of non-traumatic cerebral hemorrhage. The chest CT was performed at 1 week and 6 months after surgery. Three-dimensional (3D) reconstruction was performed using chest image process (CIP, a 3D slicer extension program) for quantitative evaluation of volume and mass of lung.

Results: The volume and mass at postoperative 1 week CT accounted for $27.7 \%, 35.45 \%$ in the right lower lobe (RLL) and $29.79 \%$, $27.04 \%$ in the right middle lobe (RML), respectively. Considering the datum of volume and mass, the right deviation of the heart by situs inversus totalis collapses the RLL and subsequently over-expands the RML in the early postoperative period. The mass ratio of chest CT was similar for 6 months, but the ratio of volume increased from $27.70 \%$ to $30.43 \%$ in the RLL and decreased from $29.70 \%$ to $26.80 \%$ in the RML. As time goes, the atelectasis in RLL disappeared and the volume of RLL was increased, but it did not increase to the normal size. And the hyper-expanded RML showed the tendency to change to the normal size as time goes on.

Conclusions: lung transplantation in patients with KS is considered a sufficiently challenging operation.

Corresponding author: Do Hyung Kim

E-mail:yumccs@nate.com

(c) The Korean Society for Transplantation

This is an Open Access article distributed under the terms of the Creative Commons Attribution Non-Commercial License (http://creativecommons.org/licenses/by-nc/4.0/) which permits unrestricted non-commercial use, distribution, and reproduction in any medium, provided the original work is properly cited 